\begin{tabular}{|l|l|}
\hline $\begin{array}{l}\text { Instituto de } \\
\text { Geriatria e Gerontologia }\end{array}$ & $\begin{array}{l}\text { Pan American Journal of Aging Research } \\
\text { PAJAR, Porto Alegre, v. 8, p. 1-8, jan.-dez. } 2020 \\
\text { ISSN-L: 2357-9641 }\end{array}$ \\
\hline (di) $h$ ttp://dx.doi.org/10.15448/2357-9641.2020.1.34953 & \\
\cline { 1 - 2 }
\end{tabular}

ARTIGO ORIGINAL

\title{
Efeitos do Método Pilates no equilíbrio, na força muscular e flexibilidade em idosas
}

\author{
Effects of the pilates Method in balance, muscle strength and flexibility in elderly women \\ Efectos del Método pilates en el equilibrio, en la fuerza muscular y flexibilidad en idosas
}

\section{Mariana Barcelos \\ Fabrício dos Santos ${ }^{1}$ \\ orcid.org/0000-0003-0588-0548 \\ mari.barcelos01@gmail.com}

\section{Isabela Mascarenhas de Oliveira ${ }^{1}$}

orcid.org/0000-0002-1841-803X isabela oliveiira@hotmail.com

\section{Mateus Dias Antunes 2}

orcid.org/0000-0002-2325-2548

mateusantunes@usp.br

\section{Sonia Maria Marques}

Gomes Bertolini ${ }^{1}$

orcid.org/0000-0003-2579-7362 sonia.bertolini@unicesumar.edu.br

\section{Fernanda Shizue}

\section{Nishida $^{1}$}

orcid.org/0000-0002-5078-9017 fernanda.shizue@unicesumar.edu.br

\section{Siméia Gaspar Palácio ${ }^{1}$} orcid.org/0000-0003-1849-5614 simeia.palacio@unicesumar.edu.br

Recebido em: 23 jul. 2019 Aprovado em: 2 out. 2019 Publicado em: 03 agos. 2020.

\section{(c) (i)}

Artigo está licenciado sob forma de uma licença Creative Commons Atribuicão 4.0 Internacional.

\section{Método Pilates para idosos \\ Resumo}

Objetivos: analisar os efeitos do Método Pilates no equilibrio, na força muscular e na flexibilidade em mulheres idosas.

Método: estudo de natureza intervencional com idosas do municipio de Maringá/ PR. Foram avaliadas em relação ao equilibrio, à flexibilidade e à força muscular. Ao término da avaliação, iniciaram os atendimentos fisioterapêuticos que consistiram em 10 sessões, realizadas em grupo, com duração de 60 minutos, utilizando o Método Pilates no solo. A análise estatística foi realizada pelo teste Kolmogorov-Smirnov, seguida do teste t/Wilcoxon, e do teste t/Mann - Whitney, adotando 5\% como indice de significância.

Resultados: obteve-se melhora de todas as variáveis, sendo: $10.71 \%$, para a flexibilidade; $25,47 \%$, para o equilibrio; $46,05 \%$, para a força dos músculos abdominais: e $65,44 \%$, para os músculos extensores de tronco.

Conclusões: o Método Pilates no solo mostrou-se eficaz na melhora de todas as variáveis estudadas em mulheres idosas.

Palavras-chaves: atividade motora, envelhecimento, fisioterapia.

\section{Abstract}

Objectives: to analyze the effects of the Pilates Method on balance, muscle strength and flexibility in elderly women.

Method: interventional study with elderly women from the city of Maringá - PR They were evaluated for balance, flexibility and muscular strength. At the end of the evaluation, physical therapy sessions were started, consisting of 10 sessions, performed in a group, lasting 60 minutes, using the Pilates Method in the soil. Statistical analysis was performed by the Kolmogorov-Smirnov test, followed by the $/$ Wilcoxon test, and the t / Mann-Whitney test, adopting $5 \%$ as an index of significance. Results: improvement of all variables, being: $10.71 \%$ for flexibility, $25.47 \%$ for balance $46.05 \%$ for abdominal muscle strength and $65.44 \%$ for trunk extensor muscles. Conclusions: the Pilates Solo method proved to be effective in improving all variables studied in elderly women.

Keywords: motor activity, aging, physical therapy specialty.

\section{Resumen}

Objetivos: analizar los efectos del Método Pilates en el equilibrio, la fuerza muscular y la flexibilidad en las mujeres mayores.

Metodo: estudio de naturaleza intervencional con ancianas del municipio de Maringá - PR. Se evaluaron en relación al equilibrio, flexibilidad y fuerza muscular. Al término de la evaluación, iniciaron las atenciones fisioterapéuticas que constaron de 10 sesiones, realizadas en grupo, con duración de 60 minutos, utilizando el Método Pilates en el suelo. El análisis estadistico fue realizado por el test Kolmogorov-Smirnov, seguido del test $\mathrm{t}$ / Wilcoxon, y del test $\mathrm{t}$ / Mann Whitney, adoptando el 5\% como indice de significancia. 
Resultados: obtuvo una mejora de todas las variables, siendo: $10,71 \%$ para la flexibilidad, $25,47 \%$ para el equilibrio, 46,05\% para la fuerza de los músculos abdominales y $65,44 \%$ para los músculos extensores de tronco.

Conclusiones: el método Pilates en el suelo se mostró eficaz en la mejora de todas las variables estudiadas de mujeres ancianas.

Palabras clave: actividad motora, envejecimiento, fisioterapia.

\section{Introdução}

O envelhecimento populacional é uma das mais importantes mudanças demográficas e sociais observadas em quase todo o mundo, em decorrência da diminuição da taxa de mortalidade e fecundidade que gera um prolongamento da expectativa de vida. No Brasil, o aumento da participação da população com 60 anos ou mais de idade passou de 4\% em 1940 para 8,6\% em 2000. Além disso, o Instituto Brasileiro de Geografia e Estatistica (IBGE) estima que em 2060 existirão mais de 32,6 milhões de idosos com 60 anos ou mais. ${ }^{1}$

O envelhecimento biológico humano é dinâmico e progressivo, sendo caracterizado por involuções morfológicas e funcionais no organismo que afetam a maioria dos órgãos, os quais levam a um declínio acentuado das adaptações homeostáticas. A nova realidade epidemiológica brasileira aponta para a urgência de mudanças e de inovações nos paradigmas de atenção à saúde da população idosa, que culminam em propostas de ações diferenciadas para o idoso usufruir integralmente os anos proporcionados pelo avanço da ciência. ${ }^{2}$

Certas variáveis patogênicas, tais como: perda de peso, riscos de doenças crônicas, diminuição da massa magra, perda de força e flexibilidade, quando associadas, resultam em diminuição do equilibrio, compensações na marcha e perda da mobilidade funcional das articulações. Esses fatores prejudicam a realização das atividades de vida diária (AVD's) do idoso e, consequentemente, aumentam os riscos de quedas, comprometendo a qualidade de vida. Uma das principais mudanças biológicas e funcionais que são observadas em individuos com idades entre 30 e 80 anos é a diminuição da flexibilidade e da massa muscular em torno de $25 \%$ a $30 \%{ }^{3}$
O ganho de força em idosos permite melhoria da capacidade funcional, menor risco de limitações de mobilidade, de hospitalizações e de mortalidade. Idosas fisicamente ativas beneficiam-se com o aumento da força muscular, independentemente da prática física executada. Existem evidências de que diferentes modalidades como minitrampolim, ginástica aquática e ginástica geral de piso, melhoram o equilibrio postural desse contingente populacional. ${ }^{4}$

A amplitude de movimento na maioria das articulações é gravemente restringida quando exercícios de alongamento não são realizados regularmente. Em decorrência disso, a imobilidade do aparelho osteomuscular reduz consideravelmente a flexibilidade do idoso. A diminuição da amplitude de movimento pode envolver a deterioração da cartilagem, dos ligamentos, dos tendões, do fluido sinovial e dos músculos. ${ }^{5}$

Essas mudanças podem ser atenuadas ou até revertidas através da prática de exercicios físicos, os quais são estritamente importantes para a formação óssea, devido ao efeito da carga de sustentação do peso sobre as estruturas osteoarticulares, além da ajuda na manutenção do músculo e no controle do peso corporal, proporcionando uma melhora na amplitude de movimento das articulações. ${ }^{6}$

Os exercícios baseados no Método Pilates proporcionam mobilidade na coluna vertebral e nas articulações, além de propriocepção, equilibrio e treinamento de coordenação motora. Esse método focaliza os seus exercicios na parte central do corpo, conhecido como "powerhouse" ou "core" (centro de força corporal, área entre a base da caixa torácica e a linha que vai de um quadril ao outro) e tem como principal função a estabilização.7 Dentro do conceito ampliado de core, entende-se que os extensores de quadril compreendem a parte central do corpo. Assim, a maioria dos exercícios do Método Pilates envolve a articulação do quadril, proporcionando estabilidade na região pélvica lombar, na qual a função dos extensores de quadril é considerada essencial para a estabilidade corporal. ${ }^{8}$

Quando aplicado na população idosa, o Método 
Pilates melhora a força e a mobilidade, que geralmente estão alteradas devido à presença de doenças degenerativas, como a artrite, proporcionando ainda benefícios na manutenção da pressão arterial e na calcificação óssea. Esses benefícios foram relatados em estudos, ${ }^{9-10}$ que por meio da aplicação do método, aliada ao uso de medicação apropriada, conseguiram alterar o diagnóstico de osteoporose para osteopenia após um ano de tratamento em idoso.

Dessa forma, tendo em vista os benefícios terapêuticos relatados pela literatura com a prática de atividade física em pessoas idosas, este estudo teve como objetivo avaliar os efeitos do Método Pilates no equilibrio, na força muscular e na flexibilidade em mulheres idosas.

\section{Método}

O presente estudo de natureza intervencional foi realizado na Clínica Escola de Fisioterapia do Centro Universitário de Maringá (Unicesumar), após a aprovação do Comitê de Ética em Pesquisa (CEP) da mesma universidade, sob parecer número 1.359.841, mediante a assinatura do Termo de Consentimento Livre e Esclarecido (TCLE). A divulgação do estudo foi realizada por meio de panfletos informativos. Foram selecionadas 19 mulheres saudáveis, com idade superior a 60 anos, do município de Maringá/ PR, triadas da clínica escola de Fisioterapia da Unicesumar e da comunidade em geral. Foram excluidas do estudo: mulheres saudáveis com idade inferior a 60 anos; mulheres com alguma deficiência física informada por ela mesma; com diabetes e ou hipertensão arterial descompensada (sem o uso de medicamento); ou que realizaram exercícios físicos nas 24 horas antecedentes ao teste.

As pacientes foram submetidas à avaliação do equilibrio corporal por meio da escala de Berg. da força muscular dos abdominais por meio do protocolo de Kendall e dos extensores por meio do dinamômetro e da flexibilidade corporal através do teste sentar e alcançar.

Para a avaliação do equilibrio, foi utilizada a escala de equilibrio de Berg, com escores variando de o a 56, sendo que, quanto maior o escore, melhor o equilibrio do sujeito avaliado. Cada item da escala possuiu uma escala ordinal de 5 alternativas que variaram de 0 a 4 pontos. Para a execução do teste foi preciso utilizar um cronômetro e uma régua. Cada ponto a menos na escala correspondeu a um aumento do risco de quedas; entre os escores 56 a 54, cada ponto a menos foi associado a um aumento de 3 a $4 \%$ no risco de quedas; entre 54 e 46, a um aumento de 6 a $8 \%$ de chances, sendo que abaixo de 36 pontos o risco de quedas foi de quase $100 \%{ }^{11}$

Em seguida, as pacientes foram submetidas ao teste de avaliação da flexibilidade através do Banco de Wells, também conhecido como teste de sentar e alcançar, cujo objetivo foi medir a amplitude do alongamento da parte posterior do tronco e de membros inferiores. Para a execução do teste, a paciente foi orientada a sentar-se sobre um colchonete, realizar uma flexão do tronco sobre o quadril na máxima amplitude possivel, mantendo os membros superiores estendidos à frente com uma mão colocada sobre a outra, deslizando os dedos ao longo da régua. O procedimento foi realizado três vezes, sendo considerada a medida de flexibilidade a maior distância atingida das três tentativas. ${ }^{12}$

Quanto à força muscular, a mesma foi mensurada nos grupos musculares abdominais através da avaliação manual utilizando o protocolo proposto por Kendall, ${ }^{13}$ cujo grau de força variou seguindo a ordem de classificação crescente dos termos: grau zero; vestigial; ruim; regular; bom; normal; e acima do regular. A precisão da graduação dependeu de alguns fatores tais como: a posição estável do paciente; fixação da porção proximal do músculo que foi testada; a prescrição da posição do paciente durante o teste; e a direção e a quantidade de pressão aplicada pelo examinador. Ao final da prova muscular, os pacientes foram graduados de 1 a 5, sendo 1 o grau de menor força muscular e 5 maior grau de força muscular.

Para mensurar o grau de força no grupo muscular do tronco foi utilizado o dinamômetro marca Takei, modelo 5002. Ao realizar o teste, a paciente foi orientada a manter os pés alinhados, realizar uma flexão de quadril e do tronco e, barra 
do dinamômetro ajustada na altura dos joelhos e realizar o movimento isométrico de extensão do tronco com a máxima força possivel na sequência, realizar uma extensão do tronco, repetindo o procedimento por três vezes consecutivas. Foi considerado como resultado, o melhor valor obtido.

Ao término da avaliação, iniciou-se as 10 sessões com o Método Pilates no solo, durante três vezes semanais, com duração de 60 minutos. Após o término das sessões, as pacientes foram reavaliadas pelo mesmo avaliador que realizou a avaliação inicial.

Foram seguidos os princípios básicos pelos quais o Método Pilates é executado, são eles: concentração; controle; centragem; respiração diafragmática; leveza; precisão; força; e relaxamento; sendo que os exercícios foram adaptados às condições da paciente, e o aumento da dificuldade foi de acordo com as características e habilidades individuais. ${ }^{10} \mathrm{~A}$ respiração foi considerada como uma inspiração com o corpo parado e uma expiração durante a realização do movimento. ${ }^{17,10}$

O protocolo de tratamento foi constituido por um programa de exercícios pré-estabelecidos com progressões de carga e de número de séries e de repetições a cada três sessões. O roteiro de exercícios incluiu uma sequência de relaxamento inicial, alongamento e fortalecimento de assoalho pélvico e dos membros inferiores, seguido de fortalecimento de tronco alternando as posições: deitado em decúbito dorsal; sentado na Bola Suiça e fortalecimento da musculatura dorsal em decúbito ventral; e terminando com relaxamento.

Os alongamentos incluíram a musculatura cervical, de tronco, de cadeia posterior da coluna e dos membros inferiores. Os exercícios do Método Pilates inclusos no protocolo foram: fole; heeltap com a Bola Suiça, ponte, rolamento lateral; ondulação do corpo com a Bola Suiça e ondulação completa. Além desses, também foram realizados: abdominal fabuloso na bola Suiça; abdominal no solo com magic circle; séries Single Leg Drop; séries de leg; golfinho; e estrela e exercícios sentados na Bola Suíça para equilíbrio dinâmico.

O tempo de descanso entre as repetições e as séries foi de 1 minuto. O número séries foi 3, com 10 repetições e as cargas e intensidades foram as mesmas durante toda a intervenção.

Os dados foram analisados estatisticamente pelo teste Kolmogorov-Smirnov, utilizado para calcular a média das variáveis seguido do teste t/ Mann-Whitney, que calculou a diferença relativa entre as médias pré e pós-intervenção, obtendo resultados em porcentagem, e interpretando-os como melhora dos pacientes tratados, adotando como parâmetro o nível de significância de 5\%.

\section{Resultados}

Houve melhora estatisticamente significante da variável equilibrio ( $p<0,001)$; força muscular dos músculos abdominais ( $p<0,001)$; força muscular dos músculos extensores do tronco ( $p<0,001)$; e flexibilidade $(p<0,001)$. A porcentagem de evolução do escore de equilibrio da escala de Berg foi de $35,2 \%$ e, para a flexibilidade mensurada por meio do teste "sentar e alcançar". observou o acréscimo de 54,6\%, conforme ilustrado na Tabela 1.

TABELA 1 - Média das variáveis Flexibilidade e Equilibrio pré e pós-intervenção

\begin{tabular}{ccccc}
\hline Variável & $\begin{array}{c}\text { Pré- } \\
\text { Intervenção }\end{array}$ & $\begin{array}{c}\text { Pós- } \\
\text { Intervenção }\end{array}$ & p-valor & $\begin{array}{c}\text { Diferença Relativa das } \\
\text { médias (\%) }\end{array}$ \\
\hline Média & Média & & $\mathbf{1 0 , 7 1}$ \\
$\begin{array}{c}\text { Flexibilidade (cm) } \\
\text { Equilibrio } \\
\text { (pontos) }\end{array}$ & 19,63 & 30,34 & $<0,001$ & $\mathbf{2 5 , 4 7}$ \\
\hline
\end{tabular}

*Diferença estatisticamente significativa pelo t/Mann-Whitnney. DP=Desvio Padrão 
única paciente $(5,26 \%)$ que na pré-intervenção não conseguiu realizar o teste devido ao alto encurtamento, evoluiu de $0 \%$ (cm) para 15\% (cm), nivel considerado ainda fraco.

Em relação ao progresso da força dos músculos abdominais, foi observado um avanço percentual de 94,6\%. Já a porcentagem de evolução da força dos músculos extensores de tronco, foi de 53,0\%, conforme ilustrado na Tabela 2.

TABELA 2 - Média das variáveis: Força muscular dos abdominais e dos extensores de tronco

\begin{tabular}{|c|c|c|c|c|}
\hline Variável & $\begin{array}{c}\text { Pré- } \\
\text { Intervenção }\end{array}$ & $\begin{array}{l}\text { Pós- } \\
\text { Intervenção }\end{array}$ & $\mathrm{p}$-valor & $\begin{array}{c}\text { Diferença Relativa das } \\
\text { médias }(\%)\end{array}$ \\
\hline & Média & Média & & \\
\hline $\begin{array}{c}\text { Força Muscular Exte } \\
\text { de tronco } \\
\text { (Newtons) }\end{array}$ & 38,00 & 58,16 & $<0,001$ & 65,44 \\
\hline $\begin{array}{c}\text { Força Muscula } \\
\text { Abdominais } \\
(1-5)\end{array}$ & 1,95 & 3.79 & $<0,001$ & 46,05 \\
\hline
\end{tabular}

*Diferença estatisticamente significativa pelo t/Mann-Whitnney. DP=Desvio Padrão

Vale salientar que o melhor progresso obtido na força muscular de extensores de tronco foi evidenciado em uma paciente de 60 anos (5,26\%), que evoluiu de 12 para 41 Newtons, demonstrando um aumento de 3,41 vezes do valor inicial da força da musculatura extensora.

\section{Discussão}

No presente estudo observou-se a efetividade da intervenção do Método Pilates no solo, uma vez que houve aumento da força muscular tanto dos músculos abdominais como dos músculos extensores do tronco, assim como da flexibilidade e do equilibrio. A justificativa desse progresso pode ser atribuída a um dos principios norteadores desse método: a técnica respiratória chamada de "respiração lateral", associada à expiração máxima durante os exercícios. Esses resultados são condizentes com os achados de outros autores que relacionaram o Método Pilates com a técnica respiratória. ${ }^{14} \mathrm{~A}$ respiração é o fator primordial no início do movimento, pois fornece a organização do tronco pelo recrutamento dos músculos estabilizadores profundos da coluna e da caixa torácica, favorecendo o fortalecimento abdominal e da musculatura do tronco. ${ }^{15}$

Devido à contração ativa desses músculos, um estudo, ${ }^{16}$ observou que a técnica respiratória permite uma maior expansão torácica na inspiração, e na expiração, uma maior retração torácica, o que promove maior fortalecimento da musculatura toracoabdominal durante os exercicios. Baseando-se nos seis princípios do Método, Sacco ${ }^{17}$ descreveu seis itens: concentração, consciência, controle, "centramento", respiração e movimento harmônico, sendo trabalhados de forma dinâmica visando obter aumento de força, alongamento e flexibilidade, preocupando-se em manter as curvaturas fisiológicas do corpo e tendo o abdômen como centro de força.

Além disso, por se tratar de uma atividade que não impõe desgaste articular, o alongamento dinâmico proporcionado pelo Método Pilates tem ação mútua ao relaxar os músculos tensionados demasiadamente e fortalecer os que estão estirados, diminuindo assim, os desequilibrios musculares que ocorrem entre agonistas e antagonistas, causadores dos desvios posturais na coluna. ${ }^{18}$ Partindo dessa mesma temática, um estudo ${ }^{19}$ associou a boa flexibilidade da coluna lombar a uma menor incidência de lesões lombares crônicas. No presente estudo, observou-se o acréscimo percentual de 54,6\% no nível de flexibilidade. Esses resultados conferem com os achados de outro 
estudo ${ }^{20}$, que observou um ganho de $11,74 \mathrm{~cm}$ de flexibilidade após a intervenção do Método Pilates em mulheres de $34 \pm 11,77$ anos, realizadas por 3 vezes semanais com duração de 50 minutos.

Semelhantemente a esses achados, em um estudo, ${ }^{2}$ observou uma melhora de $80 \%$ da flexibilidade de tronco em mulheres com idade entre 25 e 68 anos, evidenciando assim, a eficácia do método. Portanto, a promoção de maiores níveis de flexibilidade ocorreu pelo emprego sistematizado de alongamentos, que estimularam a extensibilidade dos músculos, tendões e ligamentos. ${ }^{22}$ Com relação ao treinamento de força muscular dos abdominais e dos extensores de tronco, o aumento da força muscular pode ser associado ao combate da sarcopenia, uma vez que possibilitou o fortalecimento das fibras musculares aumentando a resistência muscular e aprimorando o equilibrio. ${ }^{23}$

Os resultados favoráveis do ganho de força muscular nesses músculos corroboram com os observados em um estudo ${ }^{24}$, que relacionou os beneficios do Método Pilates em distúrbios da coluna lombar. Esses autores constataram que o Método Pilates se mostrou eficiente para o fortalecimento da musculatura extensora do tronco, atenuando o desequilibrio entre os grupos musculares do tronco e dos membros inferiores, diminuindo a dor lombar.

Analogamente, em outro estudo, ${ }^{25}$ também foi identificado um aumento na resistência muscular abdominal em 21 mulheres adultas sedentárias com idade média entre 26 e 47 anos após ser realizado exercicios do Método Pilates durante 5 semanas, 3 vezes semanais. Sendo assim, dentre os beneficios relacionados ao fortalecimento da musculatura abdominal e do assoalho pélvico, foram evidenciados um aprimoramento da estabilidade corporal e a mobilidade da coluna, correlacionando o ganho de força do centro do corpo com a diminuição do risco de quedas. ${ }^{26-27}$

No presente estudo foi observada uma melhora do equilibrio conforme um outro estudo ${ }^{28}$ onde foram detectados uma diminuição no risco de quedas em idosos de idade 67,3 \pm 6.5 , de ambos os sexos, bem como do aumento do equilibrio e da força de membros inferiores, após 5 semanas de tratamento com o Método Pilates. Dessa forma, com a realização de exercicios de fortalecimento de membros inferiores, observou-se melhora do controle postural, do equilibrio, da mobilidade, da flexibilidade e no desempenho das atividades de vida diária. ${ }^{29}$

Embora os achados do presente estudo apontem importantes contribuições científicas e sociais, algumas limitações devem ser destacadas. Primeiramente, a ausência de uma randomização aleatória da amostra, bem como a ausência de um grupo controle. Como segunda limitação, o tamanho amostral pode ter sido pequeno. Dessa forma, futuras pesquisas devem replicar o estudo em amostras maiores, randomizadas e que as idosas sejam avaliadas por um avaliador cego. Também se faz necessário o acompanhamento dos efeitos da intervenção a curto, médio e longo prazo.

\section{Conclusão}

Conclui-se que o Método Pilates exerceu efeito positivo melhorando o equilibrio, a força muscular de músculos abdominais e de extensores de tronco bem como a flexibilidade de mulheres idosas. O resultado obtido é relevante por tratar-se de um grupo de maior vulnerabilidade, tanto pelo sexo quanto pelo processo de senescência. As alterações hormonais decorrentes do processo de envelhecimento influenciam tanto a composição muscular quanto óssea, especialmente, na mulher. Desse modo, esse estudo contribui ao evidenciar que o Método Pilates pode melhorar a saúde e a qualidade de vida através de maior força, flexibilidade e equilibrio, auxiliando para a manutenção da autonomia da mulher idosa.

\section{Agradecimentos}

Ao Instituto Cesumar de Ciência, Tecnologia e Inovação (lceti), Coordenação de Aperfeiçoamento de Pessoal de Nivel Superior (Capes) e Programa de Bolsas de Iniciação Cientíica da Unicesumar (Probic). 


\section{Referências}

1. IBGE - Instituto Brasileiro de Geografia e Estatistica. Projeções da população do Brasil e Unidades da Federação por sexo e idade: 2010-2060. [Capturado em 2019 Set 18]. Disponivel em: https://www.ibge. gov.br/estatisticas/sociais/populacao/9109-projecao-da-populacao.html?=\&t=resultados .

2. Moreira JO. Mudanças na percepção sobre o processo de envelhecimento: reflexões preliminares. Psicol: Teor e Pesq. 2018;28(4):451-56. https://doi. org/10.1590/S0102-37722012000400003.

3. Chagas DL, Rodrigues ALP, Brito LC, Soares, E. D. S. (2018). Relação entre o equilibrio corporal e o risco de quedas em idosos de um projeto social de Fortaleza-CE. RBPFEX. 2018:12(76):547-55.

4. Oliveira MRDE, Rubens A, Dascal JB, Teixeira DC. Effect of different types of exercise on postural balance in elderly women?: A randomized controlled trial. Arch Gerontol Geriatr. 2014:59(3):506-14. https:// doi.org/10.1016/j.archger.2014.08.009.

5. Misner JE, Massey BH, Bemben M, Going S, Patrick J. Long-Term Effects of Exercise on the Range of Motion of Aging Women. J Orthop Sports Phys Ther. 1992;16(1):3742. https://doi.org/10.2519/jospt.1992.16.1.37.

6. Silva DXS, Cardoso MDSO, Melo NSL, Morais LCD. O envelhecimento e suas implicações biopsicossociais. Rev Ciênc e Soc. 2017;1(2):113-28.

7. Carvalho CBO, Dias ALM, Caldas LRR, Carneiro-Júnior MA. O método Pilates e sua influência na capacidade funcional do idoso: uma revisão sistemática. Rev Kairós: Gerontol. 2017;20(3):223-35.

8. Curi VS, Haas AN, Alves-Vilaça J, Fernandes HM. Effects of 16-weeks of Pilates on functional autonomy and life satisfaction among elderly women J Bodyw Mov Ther. 2018;22(2):424-29. https://doi. org/10.1016/j.jbmt.2017.06.014.

9. Kopitzke R. Pilates: a fitness tool that transcends the ages. Rehab Manag, 2007;20(6):28-31.

10. Silva ACLG, Mannrich G. Pilates na reabilitação: uma revisão sistemática. Fisioter Mov. 2009;22(3):449-55.

11. Braz NFT, Dutra LR, Medeiros PES, Scianni AA, Faria CDCDM. Effectiveness of Nintendo Wii in functional and health outcomes of individuals with Parkinson's disease: a systematic review. Fisiote Pesq. 2018;25(1):100-06. https://doi.org/10.1590/1809-2950/17131825012018.

12. Fonseca AIS, Barbossa TC, Silva BKR, Ribeiro HS, Quaresma FRP, Maciel ES. Efeito de um programa de treinamento de força na aptidão física funcional e composição corporal de idosos praticantes de musculação. RBPFEX. 2018;12(76):556-563.

13. Kendall FP. Músculos: Provas e Funções. $5^{\mathrm{a} e d}$ São Paulo: Manole; 2007.

14. Balogh A. Pilates and pregnancy. RCM midwives. 2005;8(5):220-222.
15. Sousa MEB, Martins DJN, Gonzaga DB, Oliveira CAS, Magalhães GM, Bastos VPD. Influência do método pilates na função cardiorrespiratória de idosos. Rev Expressão Católica Saúde. 2018;2(1):32-41. https://doi.org/10.1590/1809-2950/17131825012018.

16. Santos M, Cancelliero-Gaiad KM, Arthuri MT. Efeito do método pilates no solo sobre parâmetros respiratórios de individuos saudáveis. Rev. bras. ciênc. mov. 2005:23(1):24-30. https://doi.org/10.1590/18092950/17131825012018.

17. Sacco ICN, Andrade MS, Souza OS, Nisiyama M, Cantuária AL, Maeda FYl, et al. Método pilates em revista:aspectos biomecânicos de movimentos específicos para a reestruturação postural - Estudos de caso. Rev. bras. ciênc. Mov. 2005:13(4):65-78.

18. Bertoli J, Dal Pupo J, Vaz MA, Detanico D, Biduski GM, Freitas CR. Effects of Mat Pilates on hip and knee isokinetic torque parameters in elderly women. J Bodyw Mov Ther. 2018;22(3):798-804. https://doi. org/10.1016/j.jbmt.2017.08.006.

19. Rosa HL, Lima JRP. Correlação entre Flexibilidade e Lombalgia em Praticantes de Pilates. Revi Min Educ Fís Viçosa. 2009:17(1):64-73.

20. Barra BS, Araújo WB. O efeito do método Pilates no ganho da flexibilidade. [monografia]. Graduação. Faculdade de Ciências Aplicadas "Sagrado Coração" UNILINHARES, Linhares, 2007.

21. Carvalho CM, Ferreira SCB, Cardoso F, Cunha MAT, Ribeiro CHV, Silva VF. A influência dos exercicios do método Pilates na flexibilidade de mulheres. EfDepoertes, 2009:14(139):1-10.

22. Branco ANC, Miyamoto GC, Soliano ACG, Farhat HA, Franco KFM, Cabral CMN. Comparison of satisfaction, motivation, flexibility and delayed onset muscle soreness between modern Pilates method and unstable Pilates method. Fisioter e Pesq. 2017:24(4), 427-36. https://doi.org/10.1590/1809-2950/17685224042017.

23.Foster C, Armstrong ME. What types of physical activities are effective in developing muscle and bone strength and balance?. J Frailty Sarcopenia Falls. 2018:3(2):58-65. https://doi.org/10.22540/JFSF-03-058.

24. Kolyniak IEGG, Cavalcanti SMB, Aoki MS. Avaliação isocinética da musculatura J Phys Ther Sci. 2014:26(4):475-77.

28. Bird ML, Fell J. Pilates Exercise has Positive Long Term Effects on the Aged-Related Decline in Balance and Strength in Older, Community Dwelling Men and Women. J Aging Phys Act. 2013;22(3):342-47. https://doi.org/10.1123/JAPA.2013-0006.

29. Silva ACLG, Mannrich G. Pilates na reabilitação: uma revisão sistemática. Fisioter. Mov. 2017;22(3):449-55.

Mariana Barcelos Fabrício dos Santos

Fisioterapeuta pelo Centro Universitário de Maringá (UNICESUMAR, Maringá, PR, Brasil). 
Isabela Mascarenhas de Oliveira

Fisioterapeuta pelo Centro Universitário de Maringá (UNICESUMAR, Maringá, PR, Brasil).

\section{Mateus Dias Antunes}

Mestre em Promoção da Saúde pelo Centro Universitário de Maringá (UNICESUMAR, Maringá, PR, Brasil), Doutorando em Ciências da Reabilitação pela Universidade de São Paulo (USP, São Paulo, SP, Brasil).

\section{Sonia Maria Marques Gomes Bertolini}

Doutora em Ciências Morfofuncionais pela Universidade de São Paulo (USP, São Paulo, SP, Brasil), Coordenadora do Programa de Pós-Graduação em Promoção da Saúde, Centro Universitário de Maringá (UNICESUMAR, Maringá, PR, Brasil).

\section{Fernanda Shizue Nishida}

Doutora em Enfermagem pela Universidade de São Paulo (USP, São Paulo, SP, Brasil), professora do Programa de Pós-Graduação em Promoção da Saúde. Centro Universitário de Maringá (UNICESUMAR, Maringá, $P R$, Brasil).

\section{Siméia Gaspar Palácio}

Doutora em Ciências Médicas pela Universidade de São Paulo (USP, São Paulo, SP, Brasil), professora do Curso de Fisioterapia, Centro Universitário de Maringá (UNICESUMAR, Maringá, PR, Brasil).

\section{Endereço para correspondência}

Mateus Dias Antunes

Universidade de São Paulo

Rua Cipotânea, 51

Vila Universitária, 05360-000

São Paulo, SP, Brasil 